\title{
Study on The Earthquake Disaster Reduction Information Management System and Its Application
}

\author{
Youhai Guan \\ China University of Petroleum/Department of Civil Engineering, Qingdao, P.R.China \\ Emai: gyhlhl@163.com \\ Xudong Cheng \\ China University of Petroleum/Department of Civil Engineering, Qingdao, P.R.China \\ Email: chengxd@upc.edu.cn \\ Yuan Zhang \\ China University of Petroleum/Department of Civil Engineering, Qingdao, P.R.China \\ Email: vnh1980@163.com
}

\begin{abstract}
It is significant to scientifically carry out the urban earthquake disaster reduction. According to the features of China urban earthquake disaster reduction, this paper designed the urban earthquake disaster reduction information management system, which proposed a system design idea, system composition and function structure. The system adopted the object-oriented language VB6.0 and the component set ArcGIS Engine provided by ESRI for development. We applied a variety of information techniques (GIS and database) for spatial information acquisition, analysis and computing, and drew up function modules corresponding to inquiry, spatial analysis, risk analysis and data management. By using this system we can achieve scientific management about the earthquake disaster information in storage and transportation engineering, draw up kinds of earthquake emergency decisions intellectually and make them visual, which improved the efficiency and velocity of earthquake emergency evidently, and assisted the decision-making system effectively for the earthquake emergency work .
\end{abstract}

Index Terms-Earthquake disaster reduction, Information system, ArcGIS Engine

\section{INTRODUCTION}

Earthquake disasters are primarily caused by destruction due to earthquake, including the damage of buildings, engineering facilities and social systems. Problems will also occur in disaster-relief-related social order and organizations when earthquakes happen. Evidence obtained both home and abroad indicates that it is vital to reduce the earthquake damage and loss by carrying out the urban earthquake disaster reduction before earthquakes take place.

Nowadays, there is a growing number of Chinese cities making the earthquake disaster reduction plan. However, taking into account the basic fact that modern and outdated architectures coexist in cities as well as the tearing down and the reconstruction of many buildings, the conventional text style method of earthquake disaster reduction is no longer capable. In order to solve this problem, this paper designed a highly universal, low development cost and easily-maintained earthquake disaster reduction information management system, which based on the advanced technique ArcGIS Engine and other popular software system component techniques. The system uses information to conduct a variety of risk analysis, figures out the weaknesses in urban earthquake disaster reduction and then work out the final disaster reduction plan.

\section{DeVElopment PlatForm}

There are three major methods for making application programs when developing the information management system. The first way is compiling the codes in the VBA environment of ArcGIS Desktop software. But it also has a serious defect, that is the source codes are saved in the templates or files, which makes it inferior in security and scalability. The second way is using the program languages which support COM technique. Compile DLL documents which can be added to the ArcGIS Desktop through specific interfaces which achieved the openness of ArcObjects. The third way is taking advantage of the visual component MapControl consisted in Arcobjects and component PageLayoutControl to develop the GIS application program which has the independent interface. However, these three development methods all require the clients to set up certain types of ArcGIS Desktop products, which leads to a rather high product arrangement cost. On the other hand, the Arcobjects package granularity is quite minute, and there are over 1000 objects in complicated organization, which is very 
difficult for practical application. Moreover, Arcobjects cannot run detaching from ArcGIS platform, so the cost will be extremely high. Since ArcGIS Engine re-packages the Arcobjects core objects, it is still very powerful. And the application program developed by $\mathrm{AE}$ can work independently on terminals without any ArcGIS so long as it obtains the software license.

The newly released ArcGIS Engine by ESRI is an embedded component system based on OCX control technique. It contains three key parts, which are control, toolbar and tool, and object library[1-2]. The control is the constituent of ArcGIS user desktop. Users can insert and use it in application programs. Toolbar is the set of GIS tools, which is used to interact with map and geographic information. Tool is presented as toolbar on application program interface. The process of application establishment has been successfully simplified by adopting a set of various common tools and toolbars. Object library is the set of program ArcObjects components, including set graphics, drafting, GIS data source, Geodatabase and other libraries. Programmers can develop all kinds of applications from lower to higher levels via these libraries in the developing environment platforms such as Windows, Unix and Linux. The same GIS library is also the base of ArcGIS Server software which consists the desktop software ArcGIS[3-4].

This system uses advanced program language VB, which is object-oriented and event-driven, as the secondary development language, and the background database development adopts ACCESS and ADO techniques.

\section{THE SYSTEM DESIGN}

\section{A The System Structure Design}

The system is composed of database, database management system, function modules (FM), and user graphical interface. The database includes spatial database and attribute database. They are connected by keywords, which can bring about the synchronous data searching and updating. The visit to database is realized by ADO technique. The database management system can manage the background ACCESS database independently without opening the map file[5-6]. Each function module adopts the component GIS technique that is the Visual Basic + ArcGIS Engine. The system also uses AE components such as MapControl 、 PageLayoutContro1、TocContro1 and ToolbarControl to accomplish the basic functions of map view, roaming, scaling, key element searching and map print. The user interface is designed by VB. System structure is shown as Figure 1.

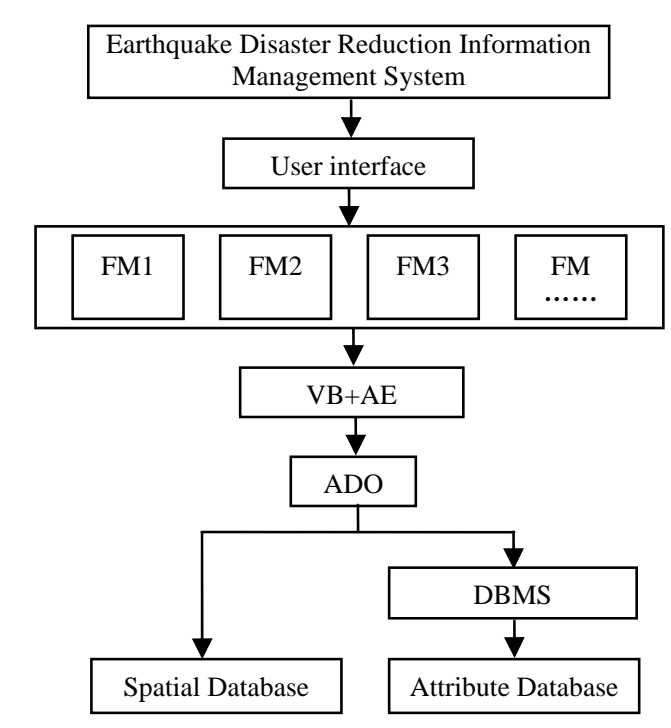

Figure 1. System structure

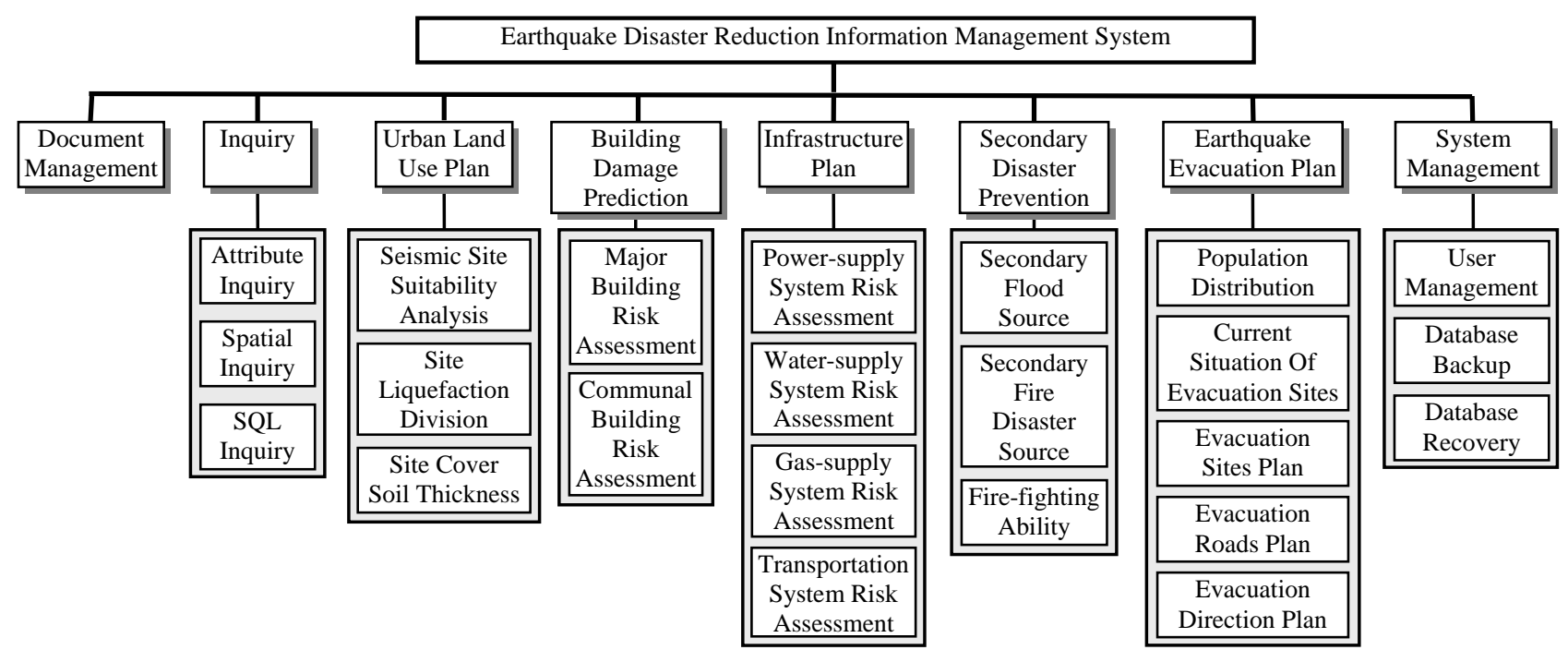

Figure 2. The structure of system function 


\section{B System Function Design}

According to the system data and function demand analysis, followed by the system design principle, we divided the system into 8 modules, which are document management, map instrument, urban land use plan, building plan, infrastructure plan, secondary disaster prevention plan, earthquake evacuation plan and system management. The structure of system function module is shown as Figure 2.

\section{System Data Organization}

System data is divided into electronic map data and metadata. They are conserved as .shp format and relational data respectively, and all the operations are completed based on these data. Shp format document is used to store the coordinate information of spatial data. The attribute information of surface features exists in the form of table in Geodatabase. They are associated by ID. We also define the numeric fields such as OBJECTID,SHAPE in Tab and Geodatabase, and use the same two numeric fields as related numeric fields.

\section{1) Spatial Database Establishment}

Spatial information includes planning background information, various architecture information, which includes both industrial and civil buildings, project networks information, including power-supply, watersupply networks and communication networks, state of special signs point, earthquake evacuation sites, distribution of command groups, hospitals, and schools, laying place of combustibles and explosive materials. Stratify and vectorise these information in ArcView, use professional seismic model to assess the risk of architectures, lifelines, sites and other systems. Then we can get the professional vector set, which can be used directly in ArcGIS Engine, an embedded GIS system. It must be noted that in the practical application lines should be connected in possible when tracing roads in ArcView and all the line targets can be traced only once. When tracing polygon targets, we must guarantee the polygon is closed in order to avoid the data loss when establishing the topological relationships. Finally it will generate the shp format document. We need to input the ID number and name of the surface feature in the dbf table of shp document. After inputting the name and the attributes, we can use the shp document directly when the mouse shows rapidly the surface feature's name, and simplify the code.

\section{2) Attribute Data}

Attribute information includes site application, building structure and seismic level, building use, roads and project networks, combustibles and explosive materials information. Set up the relational data table document based on these information and their corresponding shp document. The table has detailed attribute information about stored surface features. The system manages the attribute table data by Access database. When setting up the system, we use ADO control to call in the database document suffixes for mdb, establish the relationship between the table and the corresponding shp document, so that we are able to search the attribute and location information or use the SQL inquiry[7].

\section{3) Data access technology}

Data access technologies are ArcSDE (Spatial Data Engine) and ADO (Active Data Object)( Figure 3 (a)). ArcSDE is in charge of geographic information of SQL Server, and provides open data support for GIS software. With the structure of Client/Server, ArcSDE permits concurrent data operation of multi-user. The Client charges display of data result and the submission of user requests, ArcSDE Server takes charge of response and disposal of user request; data server charges data management. All geographic data is stored in ArcSDE Server, ArcSDE Client just puts forward request, and all of responses are finished by ArcSDE Server (Figure 3 (b)).

Every pollution source and monitor station of the coastal water accesses the system database though the Web application program developed by ASP, and realizes data input and data check in the first step. And its data access technology is ADO. ADO (Active Data Object) is the high data interface of Universal Data Access. It encapsulates OLEDB and uses COM provided by OLEDB to access SQL Server (Figure 4).

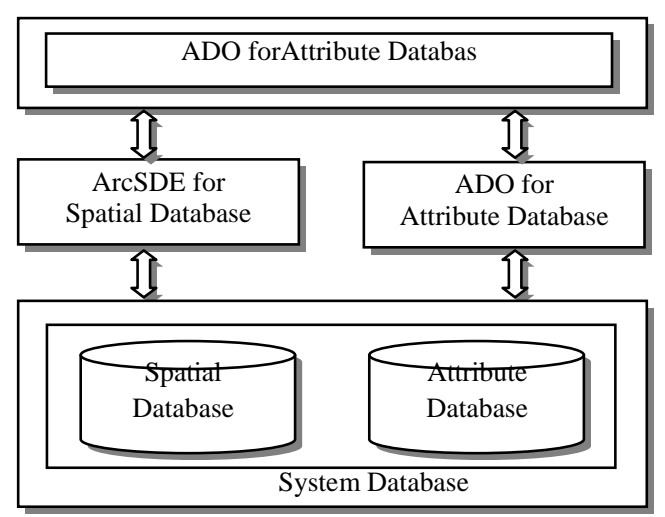

(a)

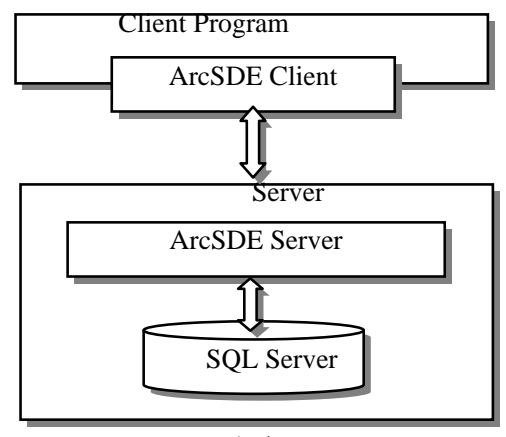

(b)

Figure 3 Data access structure 


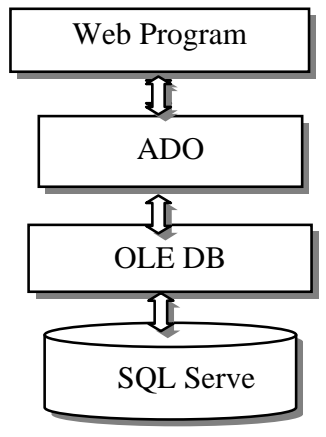

Figure 4 Data access structure

\section{4) Basic database design}

The basic database is the foundation of establishment and operation of the whole system because it combines urban basic geographical information and earthquake information in an all-round way, including the original or preliminary pretreated spatial data and attribute data each module needs to function. The spatial database in the basic database mainly stores the basic geographical electronic map in the form of layer, including: urban zonation map, building distribution map, evacuating sites distribution map, secondary disaster source distribution map, and various basic network map of lifeline projects. The attribute database in the basic database stores in the form of data table the geological background materials of the earthquake, population \& economy data, earthquake elusion \& evacuation site data, material stock data, and emergency preplan.

\section{5) Achievement database design}

The achievement database will store the result data obtained from the secondary data and model calculation, which have been dealt with by the system, so as to be utilized by relevant modules of the system. The spatial database of achievement database mainly stores different kinds of thematic maps such as thematic maps of medical demand for personnel rescue, thematic maps of allocation demand for evacuation sites, thematic maps of municipal works salvage countermeasure, thematic maps of secondary disaster defensive countermeasure. Such data as casualties' data, medical materials demand data, and materials allocation demand data will be stored in the achievement database in the form of data table.

\section{6) Data standard}

The key to making each module and sub-module of the system realize data share smoothly datum is to set up unified data standard. The data standard of the system database mainly includes: Relevant national norms such as "the product mode of the digital topographic map" (GB/T17278) and "urban geographical information system design specification” (GB/T18578) should be followed in the establishment and choice of various vector maps; as for various thematic maps generated by programs, relevant earthquake trade norms should also be followed, Such as the Code for earthquake disaster evaluation and its information management system (GB / T19428-2003), thus improving commonality and portability of the map data.

\section{SYSTEM MAIN FUNCTIONS}

\section{A Data Loading}

First we need to create a new layer file, then by using GxDialog object in the ArcGIS Engine we can unfold or conserve the geographic data within the allowable range. When using the GxDialog object, we need to set some properties first. Then we can generate a GxDataset object via DoModalOpen, add it to FeatureLayerClass, and we will obtain a shape layer.

\section{$B$ Attribute Inquiry}

Attribute inquiry allows users to search the spatial targets by the attributes. User need to set properties of the layer which needs searching first, then click on the button, the surface feature location that user finds will be highlighted on the map. At the same time, the operation information will appear in the information chart.

\section{Spatial Inquiry}

By clicking on the feature key element on the map, a dialog box which includes the attribute information will pop-up, this function achieves to complete the inquiry directly, quickly and conveniently. ArcGIS Engine provides the IdentifyDialog object, which generates relationships among record sets of MapLayer and Table. The search result contains all the attribute information of the surface feature. The shp format document after establishing the relationships is read only.

\section{SQL Comprehensive Inquiry Function}

It may not meet the requirements of users to find the location based on attribute information or do it conversely. Users can find all the surface attribute informations in which he or she is interested by inputting the search requirement. Therefore, SQL can provide more advanced search services, which improves the system value. ArcGIS Engine provides the IsqlQueryDialog object, using the data in the attribute database to find the spatial surface features locations.

\section{$E$ The design and construction of model base}

The model base is the basic guarantee of with intelligence, efficiency of practicability of this system. The content that it should include depends on the functional demand of the system.

The emergency countermeasure models fall into two major groups: calamity countermeasure model and emergency demand model. The former includes all kinds of disaster-bearing body model and secondary disasters model; the latter includes the various-emergency-teamsand-materials demand model and resources optimization model.

The model development of the EARTHQUAKE DISASTER REDUCTION INFORMATION 
MANAGEMENT SYSTEM is to separate each model from the code, and each model forms into the dynamic link library (DLL) files of its own, which is utilized according to the encapsulation feature of object-oriented design (for example, class instantiation of VB), and then forms the model base of the system.

This paper mainly develops resource-demand models whose detailed contents are shown in table 1.

\section{$F$ Interface design and module integration}

In this paper, object-oriented design is the mainly used development approach in the development of each function module. In the object-oriented development, ArcGIS adopts COM, that is the object model, so as to separate some peculiar computing methods and calculation models possessed by each function module from the program in the form of dynamic link library
(DLL) files or executable files. Through the reserved interface in the program, these external files can be accessed according to certain standards, thus increasing the flexibility of the whole program. For instance, in this paper, the compiling models DLL files or executable files used in each function model of the EARTHQUAKE DISASTER REDUCTION INFORMATION MANAGEMENT SYSTEM are utilized through the class instantiation in the code of VB. After the code of each function module is compiled, the integration debug and error handling need to be carried out on the GIS platform of ArcInfo9.2. The system interface and menu items are finished by means of attached customization function design of ArcInfo9.2, and the debugged module code is integrated into the software .

TABLE I.

REQUIRING MODAL OF EARTHQUAKE EMERGENCY COMMAND

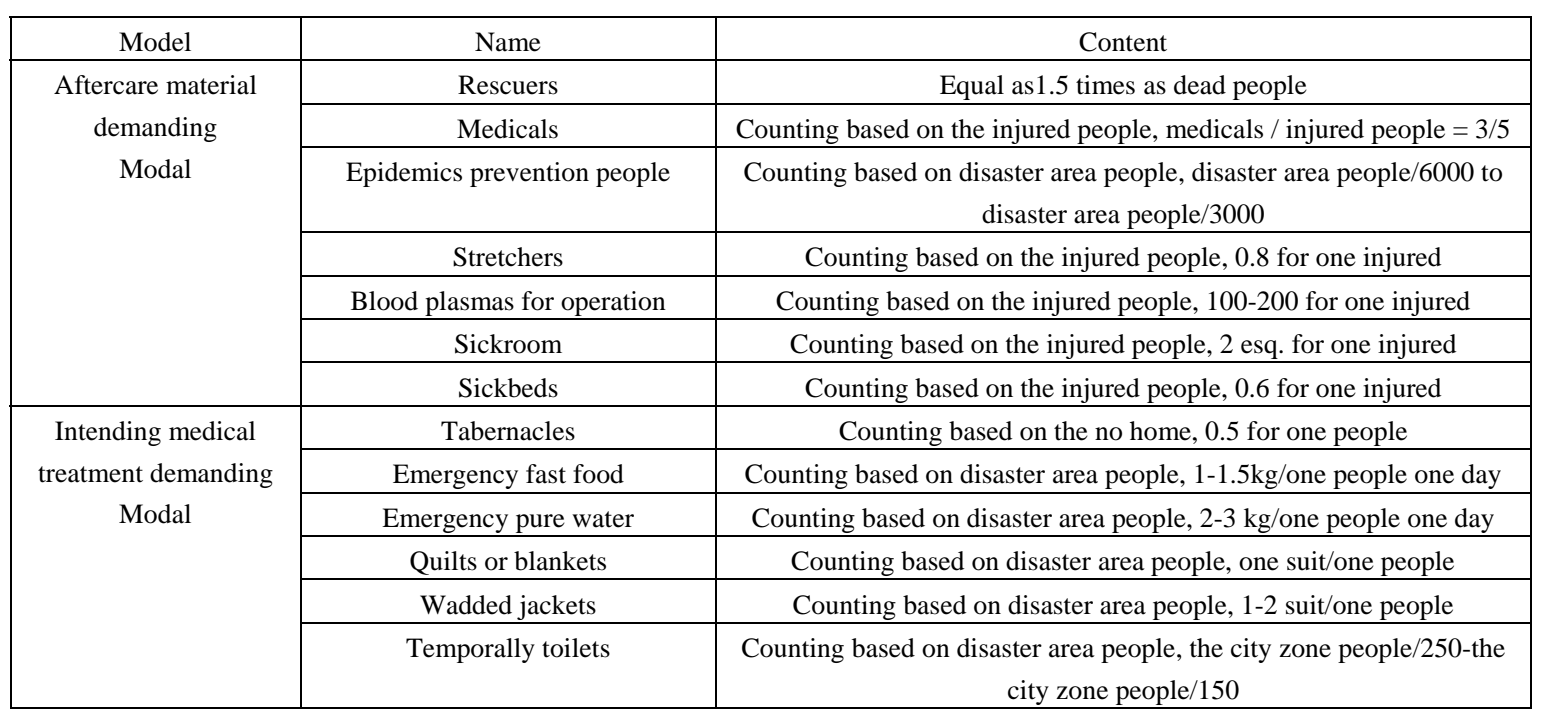

\section{EXAMPLES DEMONSTRATION}

Now let us take Zhucheng City as an illustration. Log in the system, you will enter the system operation main interface in figure 5, which includes menu bar, toolbar, spatial layer display area, status bar, station tree form search content and so forth. The spatial distribution of Zhucheng will appear on the map view, including river basin, water systems, administrative sectors, administrative center locations, all the city roads, watersupply networks, seismic weak areas, disaster prevention command and important departments, schools, public facilities, evacuation sites and geological drilling positions.

The functions of toolbar, content column and map view in the system can all use the developed components such as ToolBarControl, TOCControl, MapControl and PageLayoutControl in the embedded GIS system ArcGIS Engine. It achieves the basic GIS functions, including scaling, roaming, layer management, special subject map making and page layout. The system has integrated several seismic analysis professional models, accomplished the display of various systems seismic risk analysis. Each professional model can be called out by menu bar and toolbar. The system also has other functions such as city data statistical analysis, report forms and inquiry, which includes map inquiry, attribute inquiry and condition inquiry. Application status bar control has the functions of showing the state of present map, operation tips and copyright information.

This system can achieve the following functions:

(1) Systemize earthquake information, disaster information, emergency information and so on in order to form the information processing system which can track the seismic activity, grasp the trend, determine the disaster situation, propose the Earthquake Emergency countermeasure of storage and transportation engineering. And through information networks, transmit the relevant information to the relevant departments to form a highly integrated, fast and reliable decision support system.

(2) This system is a part of the public safety emergency command system. It can provide information 
such as earthquake situation, disaster situation and program by using VB6.0 based on ArcGIS Engine,

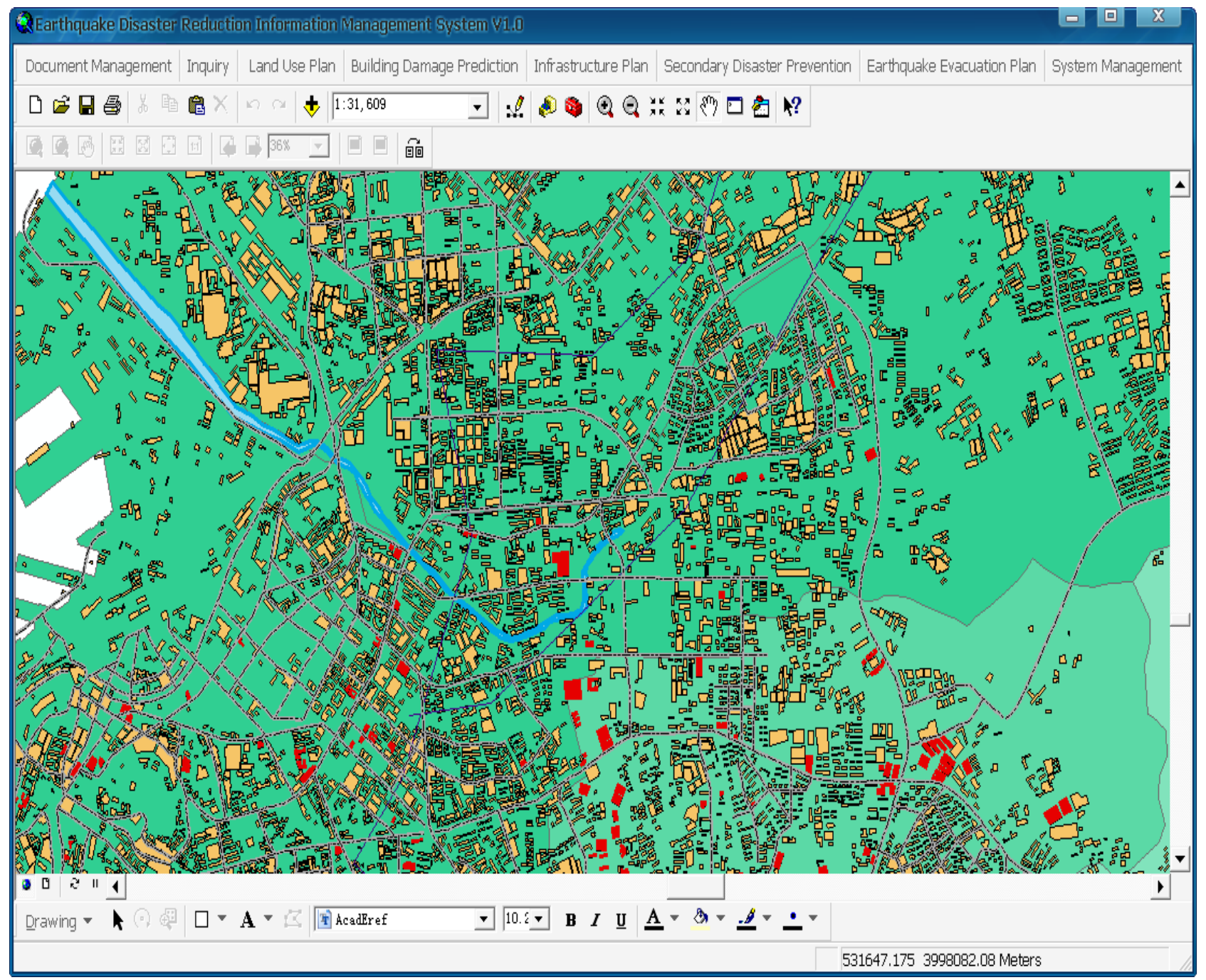

Figure 5. System main interface

countermeasures in time for the safety emergency command center.

(3) This project is very practical, and can be used in cities of oil and gas storage and transportation base or oilfield cities. This system is objective, scientific and operable, and can be able to track and grasp earthquake situation and damage situation dynamically. Besides, it has the ability to provide emergency countermeasures, and carry out search, statistics and analysis. In addition, it also has the function to update and expand itself conveniently, etc.

\section{CONCLUSIONS}

As an embedded developed product based on ArcObjects, ArcGIS Engine can completely work without ArcGIS, it also has many controls, which simplify the development even further. Thus, the developers can develop the GIS system quickly which is both powerful and meet the user requirement. This paper discussed the method of developing the application represented its advantages of low cost, integrity and efficiency.

By using this system we can achieve scientific management about the earthquake disaster information in storage and transportation engineering, draw up kinds of earthquake emergency decisions intellectually and make them visual, which improved the efficiency and velocity of earthquake emergency evidently, and assisted the decision-making system effectively for the earthquake emergency work

We developed the Zhucheng earthquake disaster reduction management information system based on ArcGIS Engine embedded GIS component technique, used data to interpret the abstract problems in the disaster reduction plan. The system not only has the common features, which are scaling, roaming, layer management and the interoperation between the map and attribute, but also adds the seismic assessment professional achievements into the system, which ensure the spatial management and analysis of various information in the plan. The establishment and operation of this system not only accomplish the conservation, inquiry, assessment 
and visual analysis management of numerous spatial and attribute data but also improve the quality and efficiency of decision-making and public service of certain departments. It is of key importance to encourage the reform of management pattern accomplish the informationization of disaster reduction[8-11].

This system also needs improving in several respects, for example, the map edit, network map analysis and application. This system only adds special subject map information of surface features, but lacks 3D dynamic image map. The attribute inquiry is still quite limited in function, which needs further dynamic update of spatial data. The data management also needs improving.

\section{ACKNOWLEDGMENT}

This research was founded by National Natural Science Foundation Project "The research of optimization method based on the urban behavior of seismic fortification standard" (ID: 50778167).

\section{REFERENCES}

[1] Zhang Bin, "GIS development technique based on ArcGlS Engine,"[J]. Graduate learned journal of Wuhan University(Natural Science), 2004, 21(3): 173-176.

[2] Xia Bo, Liu Hongbin, and Wu Wei, "The establishment of soil testing and fertilization information management system based on ArcGIS Engine,"[J]. Computer and modernization, 2007(1): 108-111.

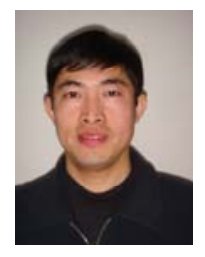

Youhai Guan was born in Pingyi, Shandong, Oct.1975. He earned Ph.D. degree in environmental engineering in Ocean University of China, Qingdao, Shandong in 2006.

Now he works as the Associate Professor in Department of Civil Engineering, China University of Petroleum in Qingdao.

His publications include: Study and application of a decision method of comprehensive disaster prevension plan for lifeline projects(Journal of Catastrophology, 2010), Study on decisionmaking of water supply pipeline comprehensive disaster prevention plan and its application(Technology for Earthquake Disaster Prevention, 2010), and Optimization of urban seismic fortification criteria(Earthquake, 2010).

Dr. Guan is the Graduate Student Tutor in Department of Civil Engineering, China University of Petroleum.
[3] Xue Wei, The design of GIS, [M]. Beijing: National Defense Industrial Press, 2004.

[4] Zhu Zheng, Application of GIS based on ArcGIS Engine, [M]. Beijing:ESRI(Beijing)Co.,Ltd, 2004.

[5] Liu Benyu and Ye Liaoyuan, The application of artificial intelligence in seismic disaster reduction projects,[M]. Beijing:China Building Industry Press, 2003.

[6] Guan Youhai, Feng Qimin, and Ma Haoran, “The design and development of seismic emergency response and decision making support system," [J], World Earthquake Engineering, 2006, 22(1):22-28.

[7] Guan Youhai, Feng Qimin and Wu Yuntao, Design and Development on Urban Earthquake Emergency Countermeasure Spatial Decision Support System, Proceedings of the 2006 International Symposium on Safety Science and Technology, Changsha, China, pp513517, Oct. 24-27, 2006.

[8] Guan Youhai and Wang Suzhen, "The development and application of Qingdao earthquake emergency command system,”[J]. 2007, Vol.1, No.1: 12-18.

[9] Jie Gao, Feng Qimin, and Shanjun Mo, "Urban earthquake disaster prediction and its general program research of information management," World Earthquake Engineering, Vol. 21, No. 1, pp.75-08, January 2005.

[10] Hong Chen and Feng Qimin, "The database design of urban earthquake emergency command decision support software,” World Earthquake Engineering, Vol. 21, No.4, pp.77-81, April 2005.

[11] Weiqin Liu and Jinghai Xu, "Urban earthquake prevention and disaster reduction information system development based on GIS," Journal of Nanjing University of Technology, Vol. 25, No.1, pp.14-18, January 2003. 